\title{
Effect of Water on Bombyx mori Regenerated Silk Fibers and Its Application in Modifying Their Mechanical Properties
}

\author{
Gustavo R. Plaza, Paola Corsini, Jose Perez-Rigueiro, Enrico Marsano, \\ Gustavo V. Guinea, Manuel Elices \\ Departamento de Ciencia de Materiales, ETSI Caminos, Canales y Puertos. Universidad Politecnica de Madrid, \\ 28040 Madrid, Spain \\ Dipartimento di Chimica e Chimica Industriale, Universita di Genova, Via Dodecaneso 31-16146 Genova, Italy
}

\begin{abstract}
The effect of water on regenerated silkworm silk fibers has been studied and compared with that of water on natural silkworm silk fibers. Regenerated fibers are spun from an N-methylmorpholine-N-oxide (NMMO) fibroin solution through a wet-spinning process, leading to fibers with two distinct tensile behaviors, labeled as brittle and ductile, respectively. Regenerated fibers show a significant contraction when immersed in water. Contraction increases further after drying. In contrast, natural silkworm silk fibers show a negligible contraction when submerged in water. Regenerated fibers tested in water are considerably more com-
\end{abstract}

\section{INTRODUCTION}

Silkworm (Bombyx mori) silk has been a valuable material in the textile industry for over 2000 years. Its use is justified both by an exceptional combination of mechanical properties in terms of strength, stiffness, and resilience, and its convenient storage in the cocoon. A single cocoon may provide over $1000 \mathrm{~m}$ of fibers by a simple process known as degumming that essentially consists of immersing the cocoon in boiling water. The degumming process eliminates the proteinaceous sericin coating that covers the fibers and maintains the structural integrity of the cocoon, so that the core of the fiber can be recovered. The core is made up of two proteins, known as heavy chain and light chain fibroin, that convey the fiber with its remarkable mechanical behavior. Besides its traditional use, the range of applications of silkworm silk is expanding mainly in pliant than samples tested in air, though their stiffness and tensile strength are significantly reduced. It has been shown that the tensile properties of brittle regenerated fibers can be modified by a wet-stretching process, which consists of deforming the fiber while immersed in water. Regenerated wet-stretched fibers always show a ductile behavior independent from their initial tensile behavior.

Key words: proteins; biopolymers; strength; silk; regenerated fibers the field of biomaterials, due to its thermal stability up to $140^{\circ} \mathrm{C}$, biodegradability, and biocompatibility. The possibility of developing new biomaterials based on silkworm silk has driven an increasing interest in the modification of the original properties and molecular configuration of fibers.

Furthermore, silkworm silk is an appropriate system to test procedures for spinning bioinspired fibers. A paradigmatic example of bioinspired fibers would be fibers based on spider silk. Production of such fibers has been proposed to exploit the outstanding properties of this material, due to the absence of a suitable source of natural material. Spinning of bioinspired spider silk fibers will require the identification of expression systems for silk proteins and, especially, the development of spinning processes.

Regenerated silk fibroin (SF) fibers are usually spun through a wet-spinning process, differing in the composition of the spinning dope. Several solvents have been successfully used including $N$ methylmorpholine-N-oxide (NMMO) hydrate.

NMMO is not toxic, and is totally recyclable and has been used for the production of high-performance cellulose fiber (Lyocell).

The spinning of SF-regenerated fibers from NMMO solutions through a dry-jet wet-spinning 
line has been described in previous works, including the initial characterization of their mechanical properties and microstructure. It was found that, albeit SF-regenerated fibers show remarkable properties comparable to other polymeric fibers in terms of tensile strength and strain at breaking, their properties lie far below those of natural silkworm silk fibers.

The mechanical characterization of natural fibers in different solvents, and especially in water, has emerged as an extremely useful tool to understand the origin of their mechanical properties. Thus, it has been found that both silkworm and spider fibers become more compliant when immersed in water. Such behavior has been explained by the melting of a hydrogen bond network, due to the presence of water molecules. Furthermore, it has also been found that the tensile properties of both fibers diverged enormously when tested in water. On the one hand, spider silk fibers undergo a supercontraction process (a reduction of more than $50 \%$ of their initial length) and show an elastomeric behavior. On the other, silkworm silk fibers show a contraction below $5 \%$ of its original length as indicated previously and a modest decrease of the mechanical properties compared with spider silk, as is confirmed in this work.

As shown later, the study of the behavior of regenerated SF fibers submerged in water clarifies some aspects of their microstructure and the relationship between microstructure and mechanical properties. Likewise, the possibility that water can be used to modify, in a predictable and reproducible way, the properties of SF regenerated fibers have been explored, following a technique initially proposed for spider silk.

\section{MATERIALS AND METHODS}

\section{Preparation of regenerated SF fibers}

The regeneration of SF fibers was carried out in three steps: dissolution of the silkworm silk reeled from the cocoon, preparation of the dope, and spinning. A detailed description can be found elsewhere. ${ }^{17}$

Bombyx mori natural fibers were kindly supplied by Dr. G. Freddi (Stazione Sperimentale per la Seta, Milan, Italy) in freeze-dried porous blocks, which were then dispersed into a commercial aqueous solution of $\mathrm{NMMO}-\mathrm{H}_{2} \mathrm{O}(50 / 50 \mathrm{w} / \mathrm{w})$. The dispersion was concentrated under vacuum at $55^{\circ} \mathrm{C}$ and subsequently heated to $110^{\circ} \mathrm{C}$ for $20 \mathrm{~min}$ in a nitrogen atmosphere with a fast energetic stirring. Air bubbles were expelled by centrifugation at high temperature. No marked polymer degradation was observed with the molecular weight of the fibroin proteins in the range of $200-220 \mathrm{kDa}$.
Regenerated SF fibers were spun at $90^{\circ} \mathrm{C}$ with a dry-jet wet-spinning line. The diameter of the spinnerette was 100 (im and the extrusion velocity $8 \mathrm{~m} /$ min, with a corresponding shear rate on the wall of $10,700 \mathrm{~s} \sim$. At the exit of the spinnerette, the fiber passed through a $10-\mathrm{mm}$ air gap followed by a coagulating ethanol bath, drawn by a first spool which imposes on the fiber a speed of $\downarrow$. The highest value of $V_{l}$ at which the fiber was not stretched is known as free rate speed and labeled $V f$. The fibers were drawn when $V_{l}$ was greater than $V f$. The extent of drawing was quantified by the ratio DRi $=W V f$, known as draw ratio during coagulation. The fiber could be stretched by a subsequent spool, which imposed a speed of $V_{2}$ on the fiber. The extent of this additional drawing was quantified by the ratio DR2 $=V 2 / V 1$, being known as postspinning draw ratio. Collected fibers were further washed in ethanol for $-48 \mathrm{~h}$ so that NMMO was completely extracted, and finally were dried under vacuum at $40^{\circ} \mathrm{C}$ for $24 \mathrm{~h}$.

\section{Mechanical characterization}

Samples were glued on perforated aluminum foil frames $^{23}$ with a $10-\mathrm{mm}$ gauge length. Tensile tests were performed in an Instron 3309-662/8501 tensile testing machine appended to an environmental chamber (Dycometal CCK-25/300). Because of the differences in cross-sectional areas of fibers spun from different processes, forces were measured either by a $10 \mathrm{~N}$ load cell with $1 \mathrm{mN}$ resolution (Instron 2530-428) or by a $100 \mathrm{mN}$ load cell with $0.1 \mathrm{mN}$ resolution (HBM 1-Q11). The elongation of the samples was measured by means of an optical extensometer (Keyence, LS7500, 30-mm range, 1-(im resolution). The tests in air were performed at $20^{\circ} \mathrm{C}$ and $35 \%$ relative humidity. Tests in water were performed after allowing the conditioning of the fiber in water for at least $1 \mathrm{~h}$ prior to testing, to keep the duration of the whole testing process limited to a reasonable time. All tests were performed at a loading speed of $0.1 \mathrm{~mm} / \mathrm{min}$, corresponding to a strain rate of $1.7 \times 10^{\prime 4} \mathrm{~s}^{-1}$.

The diameter of the as-spun fiber was measured from the two regions adjacent to the fiber to be tested. A 5-mm long piece was cut from each side, metallized with gold and observed in a scanning electron microscope (SEM; JEOL 6300). Three micrographs were taken from each piece and at least four measurements were obtained on each micrograph. The precision of the measurements was determined from the size of a pixel as 0.3 (im. The cross-sectional area of the tensile sample was calculated from the average diameter assuming a circular cross section. In addition, each tensile-tested fiber was recovered to obtain a micrograph of the fracture surface. 
The observation conditions in the SEM were $10 \mathrm{kV}$, 0.01-0.3 nA.

The cross-sectional areas were used to obtain stress-strain curves from force-displacement plots. Elastic modulus (E), breaking strength (CJ,), and elongation at break (s,,) were calculated from the stress-strain curves. The cross sections of contracted, and subsequently dried fibers, and those of fibers tested in water were calculated from the cross section of the fibers as spun assuming volume constancy.

The longitudinal contraction of the fibers produced by water has been defined as $\left(\mathrm{L}_{0}-L_{F}\right) / L_{0}$, where $L_{F}$ is the final length of the contracted fiber and $\mathrm{L}_{0}$ the initial length. The longitudinal contraction of both SF regenerated and natural forcibly silked Bombyx mori fibers has been measured in the testing machine for this work as follows: the initial length of the samples was measured by establishing the position of the crosshead corresponding to zero load on the taut fiber. After the introduction of water the crosshead was lowered, so that the fibers were maintained unrestrained and free to contract during a conditioning time of at least $1 \mathrm{~h}$. Water was removed and the specimen was allowed to dry overnight. The length after contraction was measured by determining the new position of the zero load on the taut fiber. A similar procedure was also used to establish the initial length after contraction of the samples tested in water. The contraction effect on SF-regenerated fibers was compared with that of natural Bombyx mori fibers retrieved by forced silking.

The modification of the diameter of the fibers in water was measured by placing the fiber with both ends fixed between a piece of aluminum foil and a glass plate. The constraint in the length of the fiber impedes its contracting upon addition of water, and allows separating the effect of swelling due to the presence of water molecules in the fiber from that of contraction as a result of the coiling of the molecular chains. Four micrographs from each sample were taken in an optical microscope, and at least four measurements were taken on each micrograph.

As a final point, SF-regenerated fibers were wetstretched to evaluate the effect of this process on their mechanical properties. Wet-stretching of SFregenerated fibers started with the conditioning of the samples in water for $1 \mathrm{~h}$ with their ends fixed. Then the fibers were unloaded and allowed to contract before starting the stretching step. Samples were stretched in water up to the desired strain, unloaded and kept with their ends restrained. Water was removed and the samples were allowed to dry in air at $35 \% \mathrm{RH}$ and $25^{\circ} \mathrm{C}$ for $24 \mathrm{~h}$. Tensile tests were performed on the wet-stretched fibers, as explained earlier.
TABLE I

List of the Reels and Their Characteristics

\begin{tabular}{ccccc}
\hline Reel & $\begin{array}{c}\mathrm{DR}_{2} \\
\left(V_{2} / V_{l}\right)\end{array}$ & $\begin{array}{c}\mathrm{DRj} \\
(V i / V f)\end{array}$ & Diameter $(\mathrm{nm})$ & $\begin{array}{c}\text { Tensile } \\
\text { behavior }\end{array}$ \\
\hline 1 & 1.0 & 2.0 & $88 \pm 3$ & Brittle \\
2 & 1.1 & 7.0 & $54 \pm 30$ & Brittle \\
4 & 2.0 & 17 & $28 \pm 10$ & Ductile \\
3 & 3.8 & 2.0 & $73 \pm 8$ & Ductile \\
\hline
\end{tabular}

The diameter is expressed as mean value \pm standard deviation along the whole length of the fiber, typically $1 \mathrm{~m}$. The analysis of fibers with a large standard deviation compared with the mean value can be carried out, since the variations are small along the length of the tensiletested sample and the diameter of each individual sample has been measured.

\section{RESULTS AND DISCUSSION}

The effect of drawing on the properties of SF-regenerated fibers has been studied in previous works.

These works established that the tensile behavior of SF-regenerated fibers depends only on the postspinning drawing. This dependence implies that fibers spun without postspinning drawing $\left(\mathrm{DR}_{2} \sim 1\right)$ are brittle, in contrast with fibers spun and subjected to postspinning drawing $\left(\mathrm{DR}_{2}>1.7\right)$ are ductile and show a significant increase in their tensile strength and strain at breaking. It has also been found in such studies that the properties of the fibers were independent from the value of the draw ratio during coagulation, DRi. Here, we have used regenerated SF fibers from four reeling processes, two of them corresponding to brittle fibers $\left(\mathrm{DR}_{2}=1.0,1.1\right)$ and two to ductile ones $\left(\mathrm{DR}_{2}=2.0,3.8\right)$. The list of reels is presented in Table I.

\section{Contraction}

The contraction of unrestrained silk fibers by immersion in water is a phenomenon particularly important in spider silk fibers, which can attain a maximum reduction of $60 \%$ in their original length.

The contraction of spider silk fibers is known as supercontraction. In contrast with the spider silk fibers, silkworm (Bombyx mori) silk fibers do not show a comparable supercontraction in water. ${ }^{1}$ However, it has been found that Bombyx mori silk fibers show supercontraction when immersed in concentrated salt solutions, the extent of shrinkage being dependant of temperature and time.

The extent of the contraction in water of SF-regenerated fibers has been analyzed and compared with that of forcibly silked Bombyx mori natural fibers. The contraction produced by water on both regenerated fibers and natural Bombyx mori fibers was measured at different immersion times up to 4 days. Contraction was measured with SF-regenerated samples immersed in water and with samples immersed in 


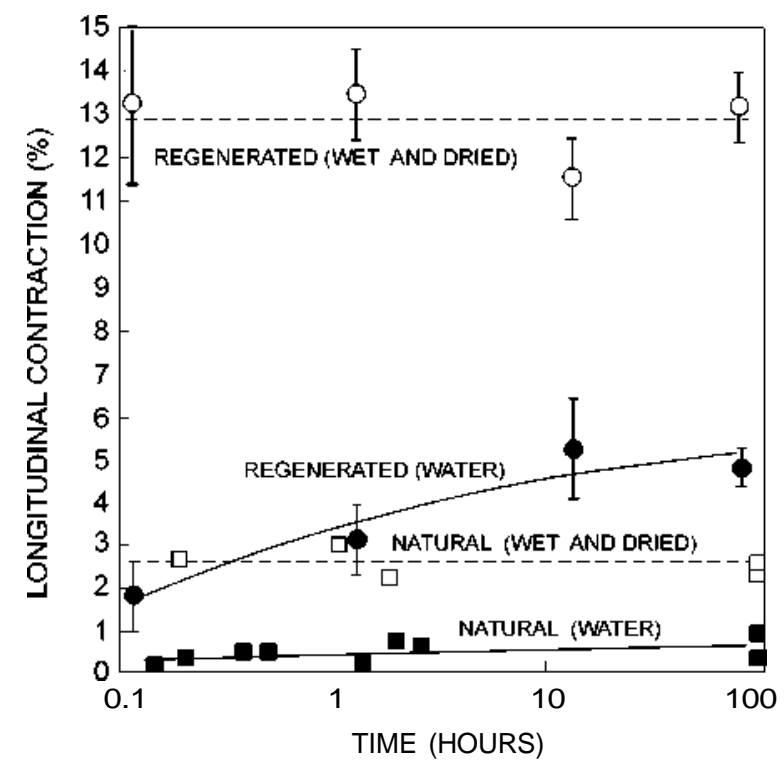

Figure 1 Contraction of SF regenerated and forcibly silked Bombyx mori fibers in water and total contraction after drying. Error bars indicate standard deviation. Time in horizontal axis is in logarithmic scale.

water and subsequently dried (drying conditions: 1 day at $35 \% \mathrm{HR}$ ), with the results being shown in Figure 1. The contraction of natural Bombyx mori fibers presents a low variability, so that each experimental point corresponds to the results of one sample. The experimental points of SF-regenerated fibers correspond to at least four measurements on different fibers due to the larger observed variability.

From Figure 1, it is evident that natural forcibly silked Bombyx mori silk shows a modest contraction in water, although contraction increases during drying. Maximum contraction of natural silk under any experimental condition is around 3\%. SF-regenerated fibers show larger contraction in water; this effect increases with immersion time. Contraction of SFregenerated fibers is even more significant after drying reaches a value of $13 \%$, which is, instead, independent of the immersion time.

Contraction is the result of two competing effects: first, molecular coiling, prompted by the increased mobility of the polymer chains due to the weakening of intermolecular interactions exerted by water; and second, the swelling, due to the inclusion of water molecules among the polymer chains. The first effect has an entropic origin and is commonly displayed by aligned polymeric materials as a result of the presence of plasticizers or variations in temperature. In particular, water breaks intermolecular hydrogen bonds both in spider and silkworm silk.

The swelling is an effect related to the volume between polymeric chains occupied by water mole- cules, which tends to increase the intermolecular distances. The larger diameter of SF-regenerated fibers when compared with natural ones offer the prospect to distinguish between both effects with a rather simple procedure.

The effect of swelling can be easily assessed from the variation of diameters when samples are immersed in water under the assumption that molecular coiling is essentially prevented in longitudinally restrained SF-regenerated fibers. The measurement of the diameter of longitudinally restrained SFregenerated fibers in water has yielded a value of $D_{\text {wa }}$ ter/Ddry $=1-24 \pm 0.02$. If the fibers are supposed to be isotropic, this ratio implies a variation in the volume due to swelling of $V_{\text {water }} / V_{d r y}=1.54$, which implies that for unrestrained samples the variation in any given direction $\left(\mathrm{V}_{\text {water }} / \mathrm{Vdry}\right)=1-15$. This value compares well with the ratio Lwater/ $/$ dried $=1.09$ obtained from measurements of longitudinal contraction (Fig. 1; the ratio between the final length of regenerated-wet and dried fibers-and the final length of regenerated water fibers). The lower value obtained for unrestrained samples is characteristic of anisotropic polymeric fibers, consistent with the anisotropy observed in SF-regenerated fibers.

\section{Tensile properties}

The influence of water on the tensile properties of regenerated SF fibers has been studied by comparing the stress-strain curves of the fibers as spun with the curves of SF-regenerated fibers contracted and tested in water, or with the curves contracted in water, dried and tested in air. At least three tests were carried out for each condition. The conditioning time in water was at least $1 \mathrm{~h}$.

Figure 2(a,b) shows the tensile stress-strain curves of brittle fibers $\left(\mathrm{DR}_{2} \sim 1.0\right)$. The curves of as-spun fibers and those contracted in water and dried are very similar. In contrast, fibers tested in water show a large increase in the strain at breaking but a significant decrease in the tensile strength.

Figure 2(c,d) shows the tensile stress-strain curves of ductile fibers $\left(\mathrm{DR}_{2}>2.0\right)$. The curves of as-spun fibers and those contracted in water and dried samples are once more very similar. Samples tested in water show a slight increase in the strain at breaking, and a large reduction in the tensile strength is apparent. The average mechanical parameters obtained from these curves are shown in Table II, where the values of natural Bombyx mori have also been included.

\section{Fractography}

The fractographic study of regenerated fibers tested as spun in air has shown that samples not subjected 

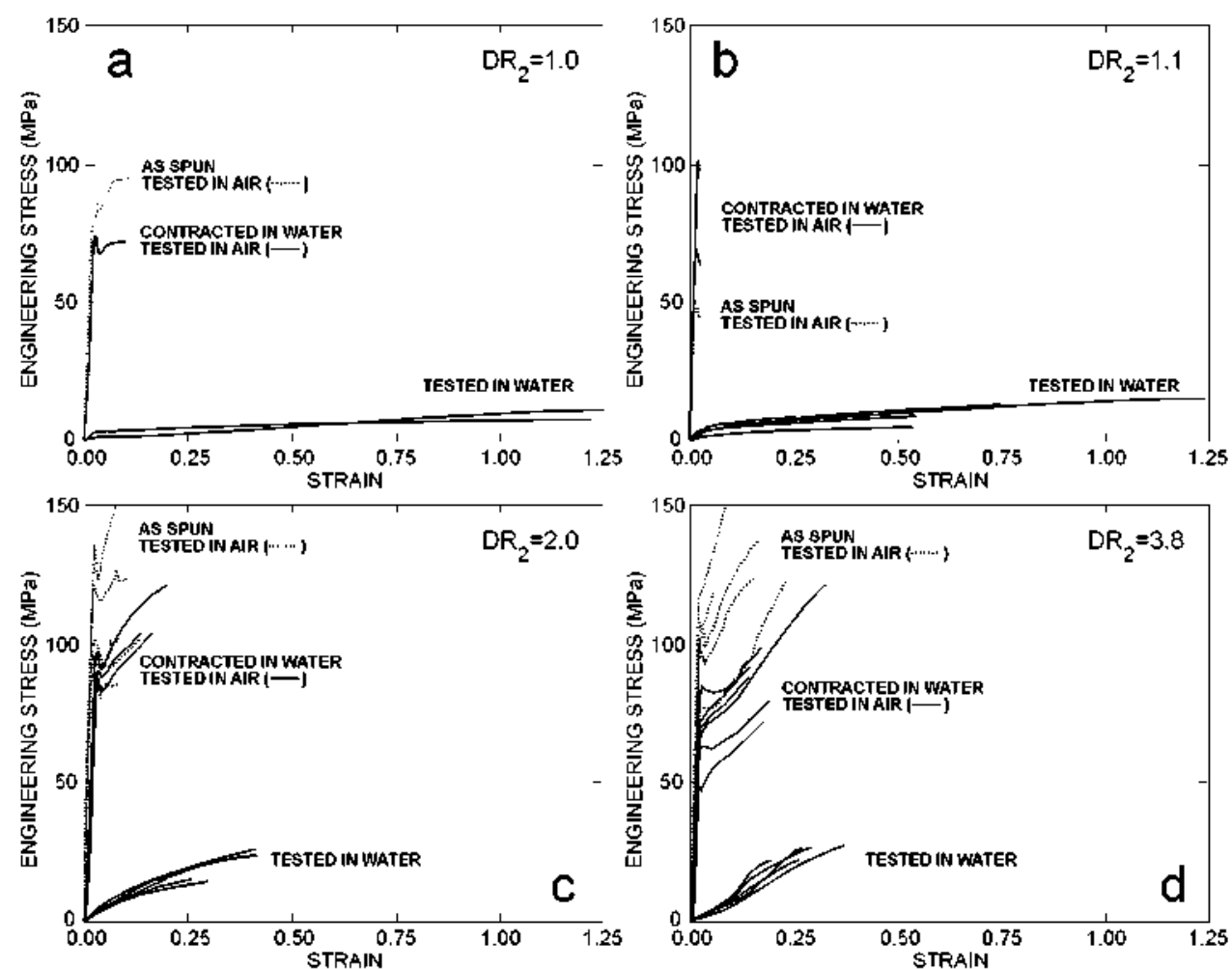

Figure 2 Stress-strain curves of regenerated SF samples tested as spun in dry conditions, tested in water and tested after drying: (a,b) low $\mathrm{DR}_{2}$ (brittle fibers), and (c,d) high $\mathrm{DR}_{2}$ (ductile fibers).

to postspinning drawing $\left(\mathrm{DR}_{2} \sim 1.0\right)$ yield a brittle behavior, which changes to ductile if a postspinning drawing $\left(\mathrm{DR}_{2}>1.7\right)$ is applied. A thorough fractographic analysis was undertaken to investigate microscopic changes produced by immersion in water on the fracture behavior.

Figure 3 compares the fracture surfaces of brittle (a,c,e) and ductile (b,d,f) SF-regenerated fibers tested in air, in water, and submerged in water, and dried and tested in air. Figure $3(a, b)$ shows the fracture surfaces of as-spun fibers tested in air and show the fractographic features described previously. Brittle fibers show mirror-like regions and flat facets, related with the absence of energy dissipating mechanisms that can prevent the propagation of cracks. In contrast, ductile fibers show a granular microstructure typical of materials with high plastic microdeformation, and characteristic of fibers spun from solution. The cause of the granular microstructure is attributed to the presence of multiple weak places originated by the voids left by the solvent during coagulation of the fibers. These voids collapse on drying and are elongated during drawing.

Figure 3(c,d) shows the fracture surfaces of fibers contracted in water, dried and tested in air, with it being apparent that immersion in water and drying do not modify the essential fractographic features observed in as-spun fibers.

Figure 3(e,f) shows the fracture surfaces of fibers tested in water. Both samples show similar fracture surfaces independently from their tensile behavior prior to immersion in water. The fracture surfaces show that the crack has grown not only perpendicu-

TABLE II

Tensile Parameters of NMMO-Regenerated Bombyx mori Silk Fibers and of Natural Bombyx mori Silk Fibers

\begin{tabular}{|c|c|c|c|c|c|c|c|c|}
\hline \multirow[b]{2}{*}{ Reel } & \multirow{2}{*}{$\begin{array}{c}\mathrm{DR}_{2} \\
\left(\mathrm{~V}_{2} / \mathrm{Vi}\right)\end{array}$} & \multirow{2}{*}{$\begin{array}{c}\text { Tensile } \\
\text { behavior }\end{array}$} & \multicolumn{3}{|c|}{ Dry } & \multicolumn{3}{|c|}{ In water } \\
\hline & & & $\mathrm{E}(\mathrm{GPa})$ & ${ }^{\circ} u(\mathrm{MPa})$ & $\mathfrak{f},(\%)$ & $£(\mathrm{GPa})$ & ${ }^{\circ} u(\mathrm{MPa})$ & $\mathfrak{f ,},(\%)$ \\
\hline 1 & 1.0 & Brittle & $3.8 \pm 0.4$ & $43 \pm 6$ & $1.1 \pm 0.1$ & $0.09 \pm 0.03$ & $10 \pm 1$ & $124 \pm 2$ \\
\hline 2 & 1.1 & & $4.2 \pm 0.1$ & $38 \pm 1$ & $1.2 \pm 0.1$ & $0.14 \pm 0.03$ & $10 \pm 2$ & $70 \pm 14$ \\
\hline 3 & 2.0 & Ductile & $7.2 \pm 0.1$ & $120 \pm 10$ & $8.6 \pm 1.2$ & $0.10 \pm 0.01$ & $25 \pm 1$ & $31 \pm 5$ \\
\hline 4 & 3.8 & & $5.3 \pm 0.2$ & $127 \pm 8$ & $12 \pm 2$ & $0.07 \pm 0.01$ & $25 \pm 1$ & $28 \pm 3$ \\
\hline \multicolumn{3}{|c|}{ Bombyx mori ${ }^{23 ; i 4}$} & $14 \pm 1$ & $570 \pm 60$ & $20 \pm 3$ & $3.8 \pm 0.3$ & $450 \pm 20$ & $27 \pm 3$ \\
\hline
\end{tabular}



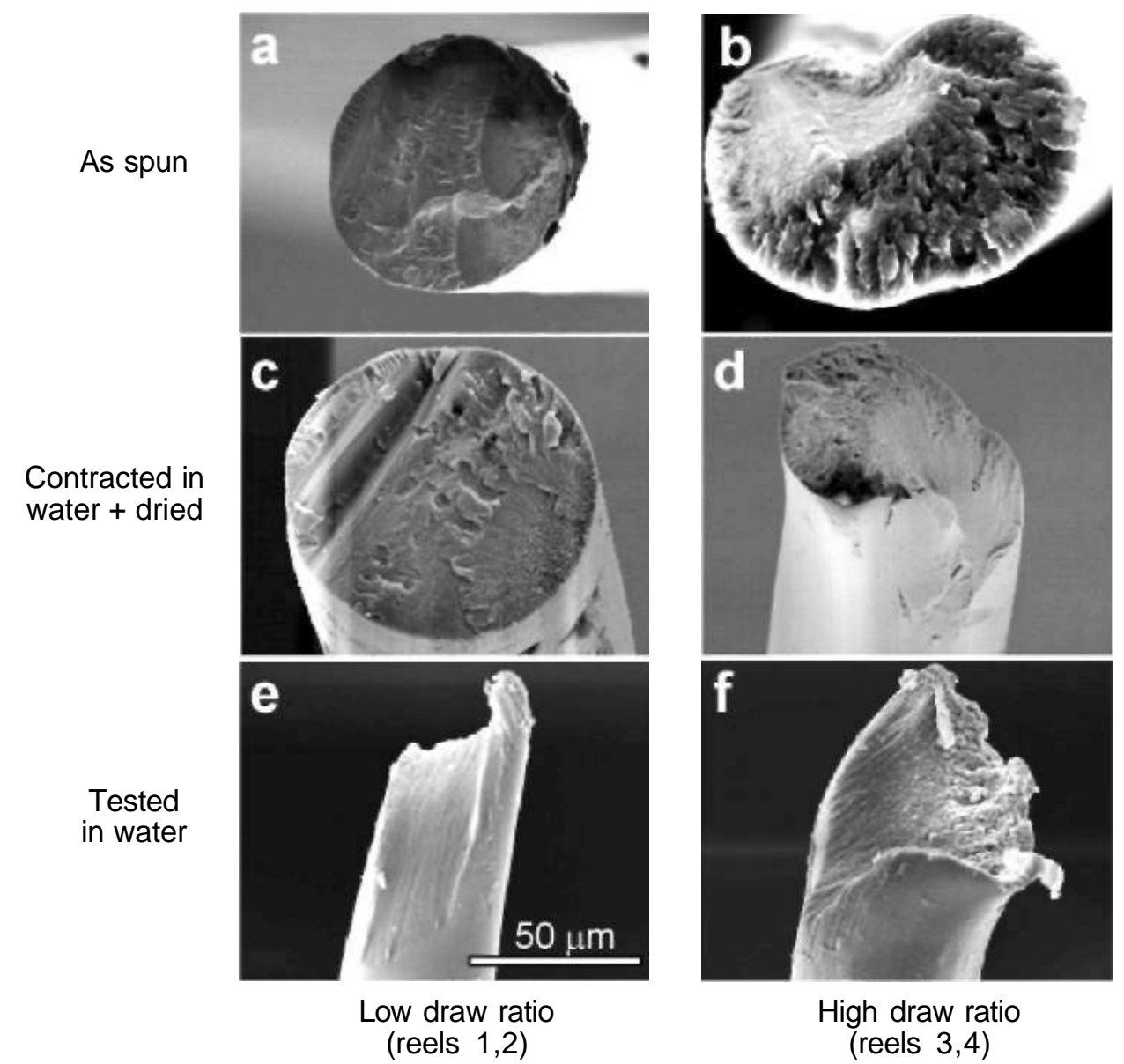

High draw ratio

(reels 3,4$)$

Figure 3 Representative fracture surfaces of the two distinct mechanical behaviors of regenerated fibers. Photographs a, c, e correspond to low $\mathrm{DR}_{2}$ fibers (reels one and two) and photographs b, d, f correspond to high $\mathrm{DR}_{2}$ fibers (reels three and four). Samples $a$ and $b$ were tested as spun, samples $c$ and $d$ were tested contracted in water and dried, and samples $\mathrm{e}$ and $\mathrm{f}$ were tested in water. All micrographs were taken at the same magnification.

lar to the axis of the fiber, but also in a parallel direction, with some features compatible with tearing.

\section{Modification of mechanical properties by wet-stretching}

The immersion in water to modify the mechanical behavior of silk fibers has led to the wet-stretching procedure, the one which allows the predictable and reproducible modification of the spider silk fibers. The wet-stretching process starts from the rubbery state induced by immersion spider silk fibers in water, aligns the chains applying a stretch in water and freezes the desired conformation by removing water, so that the hydrogen bond network is re-established.

The presence of a significant contraction effect in SF-regenerated fibers immersed in water and the influence of water in their tensile behavior suggests the possibility to modify the properties of SF-regenerated fibers through a wet-stretching process
(Fig. 4). Fibers are conditioned in water for $1 \mathrm{~h}$ and subsequently stretched up to the desired strain. Fibers are unloaded and dried for $24 \mathrm{~h}$ under conditions at $25^{\circ} \mathrm{C}$ and $35 \% \mathrm{HR}$. Figure 4 shows the stress-strain curves of three reference samples of brittle tensile behavior and the effect of the wetstretching process on two additional brittle samples. The load history during wet-stretching is shown as dashed lines. True stress (force divided by instant cross section)-strain (increment in length divided by initial length) curves have been represented to allow comparison of samples with significant differences in the strains sustained. The instant cross section is calculated from the measured initial cross section under the hypothesis of volume constancy, commonly accepted for silk fibers and proved for spider silk fibers.

Figure 4 shows that the wet-stretching process gives the initially brittle fibers $\left(\mathrm{DR}_{2} \sim 1.0\right)$ the properties of ductile fibers $\left(\mathrm{DR}_{2}>2.0\right)$. However, the properties of wet-stretched fibers do not improve those of initially ductile fibers as observed by the 

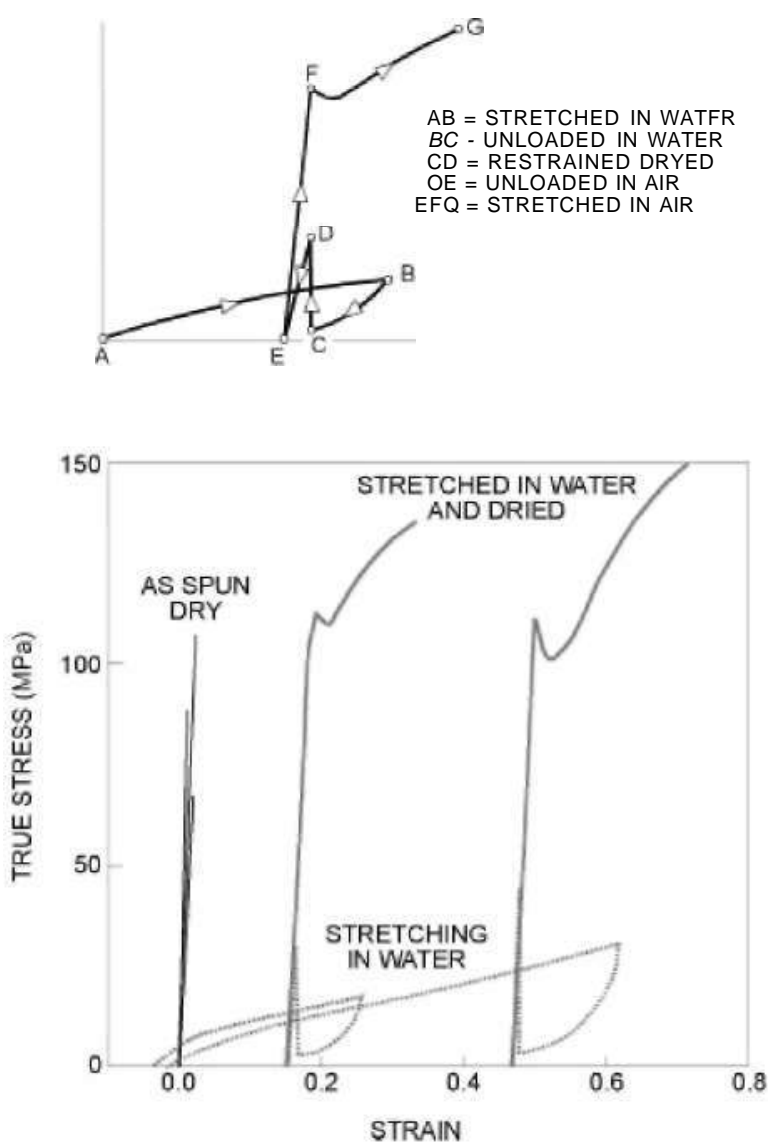

Figure 4 Stress-strain curves of regenerated SF fibers. Solid lines show the curves for samples tested as spun and tested after stretching in water. Strain is calculated as $(\mathrm{L}-$ $L o) / L o, L_{0}$ being the initial length of the fiber as spun; and true stress is calculated as $F / A, F$ being the force and $A$ the instantaneous cross section of the fiber, obtained assuming volume constancy, $A=A_{Q} L_{Q} / L$. Dashed lines show the stretching process.

comparison of Figures 4 and 2(c,d). Ductile fibers subjected to wet-stretching do not show any improvement in their initial tensile properties.

\section{Comparison of the mechanical behavior of SF regenerated and natural fibers}

One of the goals of spinning SF-regenerated fibers is the production of fibers with tensile properties comparable with those of the natural material. Figure 5 compares the range of properties exhibited by natural and SF-regenerated fibers both tested in air and in water. The elastic modulus $E$, stress at break cr,, and strain at break e,, are presented in Table II. It is apparent that the tensile properties of regenerated fibers lie far below those of the natural material.

Water induces distinct changes in natural and SFregenerated fibers. Natural fibers are slightly affected by testing in water. A reduction of the elastic modulus to $1 / 4$ of its initial value, a slight reduction of the tensile strength, and no difference in the strain at breaking can be observed. In contrast, testing in water of SF-regenerated fibers leads to a reduction of the elastic modulus to $1 / 50$ and a reduction of the tensile strength to 1/5-1/4 compared with the values of the samples as spun. Additionally, the strain at breaking of regenerated fibers is affected by the water, this phenomena being more evident for brittle fibers, which shows 100-fold increase.

The likely origin of these two distinctive behavior can be discussed from the differences in the microstructure of the natural and SF-regenerated fibers, considering that water molecules only affect the amorphous regions, leaving the microcrystals unchanged. It has been found that both natural and SF-regenerated fibers have a similar volume fraction of microcrystallites close to 0.61 , and the main difference between both materials is the degree of molecular orientation as measured by Raman spectroscopy or birefringence, SF-regenerated fibers spun at low $\mathrm{DR}_{2}$ are almost isotropic, while those spun at high $\mathrm{DR}_{2}\left(\mathrm{DR}_{2}>2.0\right)$ show a slight anisotropy, although far below the values of the natural fibers.

Consequently, the alignment of the protein chains with the macroscopic axis of the fibers appears to be related to the excellent properties of the natural silk. This hypothesis can be inferred by comparing the microstructural and mechanical data of natural and SF-regenerated fibers, but also from the modifications in the properties of silkworm silk supercontracted in concentrated salt solutions. Supercontracted silkworm silk is less deformable and shows a reduced tensile strength when compared with the properties of the fiber prior to supercontraction.

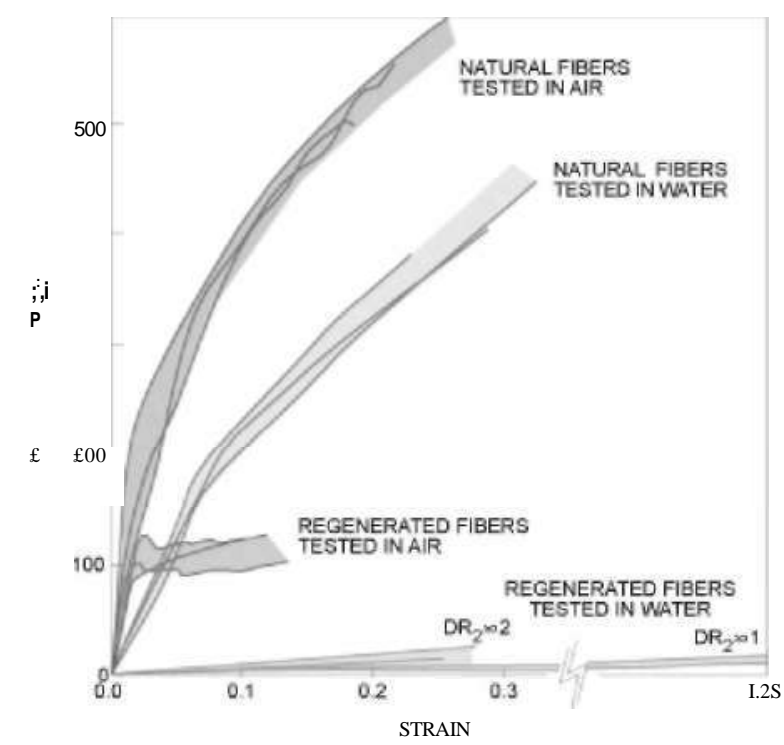

Figure 5 Range of stress-strain curves spanned by forcibly natural Bombyx mori silk fibers fibers. 
In this context, the improved properties of regenerated ductile fibers can be explained by the alignment imposed to the fiber during the postspinning drawing step. The alignment of the chains requires that the chains are able to slip against two different barriers: the weak interactions with other chains and the entanglements. The results obtained from SFregenerated fibers tested in water indicate that the slip ability of brittle fibers is increased when immersed in water. Consistent with the discussion on spider and silkworm silk, it can be hypothesized that water melts a network of hydrogen bonds. Slippage of the chains can proceed and the alignment can be frozen by removing water and re-establishing the hydrogen bonds, as demonstrated by the effect of the wet-stretching process on brittle fibers. The significant decrease in the elastic modulus and tensile strength of SF-regenerated fibers immersed in water, compared with the samples tested in air can be assigned to a greater influence of the weak interactions in the properties of regenerated fibers compared with the natural ones.

However, the alignment of the chains in SF-regenerated fibers cannot proceed up to a value comparable with natural silk fibers. The existence of this limit is evident from the similar properties that can be reached either by imposing a postspinning drawing on the fiber or by using the wet-stretching process. Since this limit must be related to a mechanism that prevents the chains from further slippage, and weak interactions are eliminated by immersion in water, it can be hypothesized that the number of entanglements is larger in regenerated fibers than in natural silk.

\section{CONCLUSIONS}

Water acts as a plasticizer in silk fibers. Its effect is markedly more important in NMMO-regenerated SF fibers than in natural Bombyx mori silk fibers. The former undergo a substantial contraction when immersed in water, i.e., five times greater than that of natural fibers obtained by forced silking. Furthermore, the tensile behavior of SF-regenerated fibers is largely affected by water. It has been found that immersion in water reduces the elastic modulus to $1 / 50$ and the rupture stress to $1 / 4-1 / 5$ of their corresponding values in dry conditions. Contrarily, the elastic modulus of natural Bombyx mori silk fibers is reduced by a factor $1 / 4$ and stress at break is hardly affected. The influence of water is of paramount importance when considering the possibility of using regenerated fibers in humid environments, as is the case in biomedical applications.

SF-regenerated fibers spun at DR2 1.0 show a brittle behavior when tested in air. This work shows that these fibers can be transformed into ductile fibers through a wet-stretching process in water. Such a method does not allow attaining the properties of natural Bombyx mori silk fibers; however, the procedure appears as a promising result and highlights the relevance of further studies to develop processes which improve the alignment of the molecular chains, so that its mechanical behavior approaches that of the natural silk fibers.

The authors thank Mario Traverso for his helpful work on the wet-spinning line, Jose Miguel Martinez for his help with drawing figures, in addition, Dott. G.Freddi (Stazione Sperimentale per la Seta, Milan, Italy) for insightful discussion.

\section{References}

Kaplan, D. L.; Lombardi, S. J.; Muller, W. S.; Fossey, S. A. In Biomaterials: Novel Materials from Biological Sources; Byrom, D., Ed.; Stockton Press: New York, 1991; p 1.

Perez-Rigueiro, ].; Viney, C; Llorca, ].; Elices, M. I Appl Polym Sci 1998, 70, 2439

Asakura, T.; Kaplan, D. L. Encyclopedia Agric Sci 1994, 4, 1.

Nakamura, S.; Magoshi, ].; Magoshi, Y. In Silk Polymers; Kaplan, D.; Adams, W. W.; Farmer, B.; Viney, $C$, Eds.; American Chemical Society: Washington, 1994; p 211.

Li, M.; Ogiso, M.; Minoura, N. Biomaterials 2003, 24, 357.

Arai, T.; Freddi, G.; Innocentri, R.; Tsukada, M. I Appl Polym Sci 2004, 91, 2383

Altaian, G. H.; Diaz, F.; lakuba, C.; Calabro, T.; Horan, R. L.; Chen, ].; Lu, H.; Richmond, ].; Kaplan, D. L. Biomaterials 2003, 24, 401.

Panilaitis, B.; Altman, G. H.; Chen, ].; lin, H. ].; Karageorgiou, V.; Kaplan, D. L. Biomaterials 2003, 24, 3079.

Minoura, N.; Tsukada, M.; Natura, M. Biomaterials 1990, 11, 430.

Sofia, S.; McCarthy, M. B.; Gronowicz, G.; Kaplan, D. L. I Biomed Mater Res 2001, 54, 139.

Nazarov, R.; lin, H. J.; Kaplan, D. L. Biomacromolecules 2004, 5, 718 .

Hofmann, S.; Hagenmuller, H.; Koch, A. M.; Muller, R.; Vunjak-Novakovic, G.; Kaplan, D. L.; Merkle, H. P.; Meinel, L. Biomaterials 2007, 28, 1152.

Fahnestock, S. R.; Bedzyk, L. A. Appl Microbiol Biotechnol 1997, 47, 33

O'Brien, I. P.; Fahnestock, S. R.; Termonia, Y.; Gardner, K. H. Adv Mater 1998, 10, 1185.

Scheller, ].; Grosse, F.; Conrad, U.; Guhrs, K. H. Nature Biotechnol 2001, 19, 573 .

Lazaris, A.; Arcidiacono, S.; Huang, Y.; Zhou, I. F.; Duguay, F.; Chretien, N.; Welsh, E. A.; Soares, I. W.; Karatzas, C. N. Science 2002, 295, 472.

Corsini, P.; Perez-Rigueiro, ].; Guinea, G. V.; Plaza, G. R.; Elices, M.; Marsano, E.; Carnasciali, M. M.; Freddi, G. I Polym Sci Part B: Polym Phys 2007, 45, 786.

Xu, Y.; Zhang, Y.; Shao, H.; Hu, X. Int I Biol Macromol 2005, $35,155$.

Marsano, E.; Corsini, P.; Arosio, C; Boschi, A.; Mormino, M.; Freddi, G. Int I Biol Macromol 2005, 34, 179.

Meister, G.; Wechsler, M. Biodegradation 1998, 9, 91.

Fink, H. P.; Weigel, P.; Purz, H. ].; Ganster, I. Prog Polym Sci 2001, 26, 1473 .

Gosline, I. M.; Denny, M. W.; DeMont, M. E. Nature 1984, 309, 551.

Perez-Rigueiro, ].; Viney, C; Llorca, ].; Elices, M. Polymer $2000,48,8433$. 
Work, R. W. Text Res J 1977, 47, 650.

Guinea, G. V.; Elices, M.; Perez-Rigueiro, J.; Plaza, G. R. J Exp Biol 2005, 208, 25

Guinea, G. V.; Elices, M.; Perez-Rigueiro, J.; Plaza, G. Polymer 2003, 44, 5785 .

Guinea, G. V.; Perez-Rigueiro, J.; Plaza, G. R.; Elices, M. Biomacromolecules 2006, 7, 2173.

Perez-Rigueiro, J.; Elices, M.; Llorca, J.; Viney, C. J Appl Polym Sci 2001, 82, 1928.

Plaza, G. R.; Guinea, G. V.; Perez-Rigueiro, J.; Elices, M. J Polym Sci Part B: Polym Phys 2006, 44, 994.

Tsukada, M.; Kato, H.; Freddi, G.; Kasai, N.; Ishikawa, H.

J Appl Polym Sci 1994, 51, 619.
Riande, E.; Diaz-Calleja, R.; Prolongo, M. G.; Masegosa, R. M.; Salom, C. Polymer Viscoelasticity, Stress and Strain in Practice; Marcel Dekker: New York, 2000.

Termonia, Y. Macromolecules 1994, 27, 7378

Chawla, K. K. Fibrous Materials; Cambridge University Press: Cambridge, 1998

Gosline, J. M.; Guerette, P. A.; Ortlepp, C. S.; Savage, K. N. J Exp Biol 1999, 200, 3295.

Hearle, J. W. S. In Fibre Fracture; Elices, M.; Llorca, J., Eds.; Elsevier: Amsterdam, 2002; p 57.

Perez-Rigueiro, J.; Elices, M.; Llorca, J.; Viney, C. J Appl Polym Sci 2002, 84, 1431.

Urn, I. C; Ki, C. S.; Kweon, H. Y.; Lee, K. G.; Ihm, D. W.; Park, Y. H. Int J Biol Macromol 2004, 34, 107. 